\title{
Design of A Smart Monitoring and Control System for Aquaponics Based on OpenWrt
}

\author{
Dan Wang ${ }^{1, \text { a }}$, Jinling Zhao ${ }^{1, b^{*}}$, Linsheng Huang ${ }^{1, c}$, Deheng $\mathrm{Xu}^{1, \mathrm{~d}}$ \\ ${ }^{1}$ School of Electronics and Information Engineering, Anhui University, Hefei, 230601, China \\ aemail: 18297955876@163.com, ${ }^{b}$ email: aling0123@163.com, cemail: \\ linsheng0808@163.com, demail: 18756955402@163.com
}

\section{Keywords: OpenWrt; environment monitoring system; remote control; Aquaponics}

\begin{abstract}
Along with the socio-economic development, more high living standards have being pursued by human beings. However, more time has been spent engaging in work and they have no extra time to look after their playthings (e.g., fishes, flowers). The primary objective is to design a smart monitoring and control device for aquaponics, which is an eco-friendly system for ornamental fish and hydroponic plants. The significance and specific functions of the system was firstly described in details, and then the architecture, hardware components and software design were also introduced. The system consists of three parts: data acquisition, mobile transfer and intelligently interactive application. Specifically, the data that acquired by webcam and some smart sensors are analyzed and processed for man-machine interaction. Meanwhile, users can also use the mobile terminal to monitor and control the smart aquaponics remotely. The design of the system can promote the rapid development of smart aquaponics.
\end{abstract}

\section{Introduction}

With the advances in technology and the improvement of people's living standards, ornamental fish and hydroponic plants become an integral part of daily life ${ }^{[1]}$. Ornamental fish and hydroponic plants not only decorate the environment, but also can delight us. However, traditional plant cultivation has been mainly performed in the soil. It is known that a series of drawbacks can be found for such a way. For example, regular watering and fertilizing have to require more time and labour $^{[2]}$. With the development of various techniques, the soilless cultivation has become a more mature and popular breeding choice such as aquaponics ${ }^{[3]}$. Aquaponics is an eco-friendly system to cultivate fish and crop without soil by utilizing aquaculture and hydroponics ${ }^{[4]}$. At present, aquaponics is mainly used in agricultural production. The balcony agriculture and family farming have more requirements for such a technique, which can not only increase the ornamental value, but also achieve an eco-friendly system. It is an inexpensive symbiotic cycle between the fish and plant. So the system can save water more efficiently.

Modern people have been always engaged in work and they have no more extra time to look after ornamental fish and hydroponic plants. On the one hand, ornamental fish conserves flings stuff and it is also tedious to replenish oxygen, maintain temperature and smears distinctly illumination. On the other hand, plants need watering and fertilization frequently. The whole process is so complex and time-consuming. Based on the above shortcomings, the automatic control system is proposed ${ }^{[5]}$. Unfortunately, current automatic control system is not stable that some unexpected errors always occur, especially it is difficult to realize remote monitoring and control. Consequently, it is very necessary to design a smart monitor and control system, especially for people who travel frequently.

Along with the rapid development of sensor technology, Internet technology, communication technology and computer technology, smart life style will become a popular trend for our future life ${ }^{[4]}$. To solve the current shortcoming, this study designs a smart monitor and control system based on OpenWrt and WRTnode, which can make it easy to implement the connection of monitoring field and remote monitoring center. The system can monitor the environment of aquaponics device through some sensors in a real-time and stable way, and then accurately and 
automatically transmit the data of temperature, humidity, light intensity, water level and photos to WRTnod in real time. WRTnode is responsible for storing and analyzing the information derived from the sensor nodes and camera. Users can view the data and photos remotely from either mobile client or web or Weibo or We chat. They can decide whether to open air pump, water pump, lights, and feeder or not based on the obtained information.

\section{Architecture of Monitoring and Control System}

The environment monitoring system is mainly made up of seven parts: aquaponics system, data acquisition, control equipment, Wi-Fi wireless data transmission module, cloud data processing server, social communication platform and mobile application. The architecture of the smart system is shown in Fig.1.

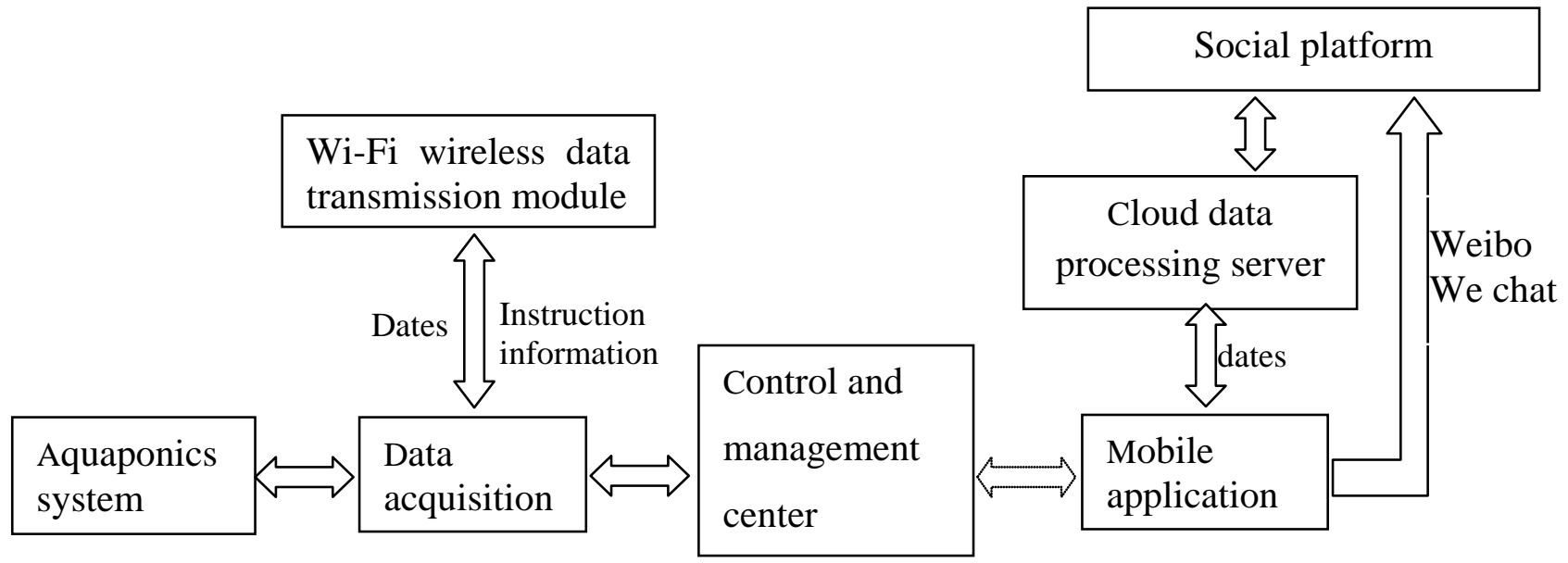

Fig.1. The overall system architecture of designed aquaponics

\section{Aquaponics system}

Aquaponics is an integrated system that links hydroponic plant production with re-circulating aquaculture. Hydroponics is a term used to describe the plants production without soil. Plant roots grow in a nutrient solution with or without an artificial medium for mechanical support ${ }^{[4,7]}$. Aquaculture, also known as aqua farming, is the farming of aquatic organisms which is the fastest growing sector of the world food economy, increasing by more than $10 \%$ per year ${ }^{[4]}$. It is an inexpensive symbiotic cycle between fish and plant. Consequently, it is necessary and significant to save energy. In our study, a compact aquaponics system was designed for household.

\section{Data acquisition}

Some sensor nodes are used to form the sensor data acquisition module, which are deployed near to the aquaponics system to collect real-time environmental information, such as temperature, humidity, light intensity, water level and photos. After an initial processing, the data is transmitted to WRTnod.

\section{Control and management center}

The control and management center is the CPU (Central Processing Unit) of the whole system, which manages all the dataset. The center consists of Arduino and WRTnod, whose primary functions are to store data from collection nodes, process data, and then upload them to the server. Another function is to download the instruction information from the server and execute.

\section{Design of the hardware system}

The hardware system consists of Arduino and WRTnod, some sensor nodes and Webcam, which is shown in Fig. 2. The system monitors the aquaponics environment through attached sensors in a 
real time and stable way, and then transmits the information to the Web server in real time, accurately and automatically. The Web server stores and analyses the information from the sensors and camera. Users can use the mobile terminal to monitor and control the smart aquaponics remotely. In other words, they make a decision whether to open air pump, water pump, lights and feeder or not based on the obtained information.

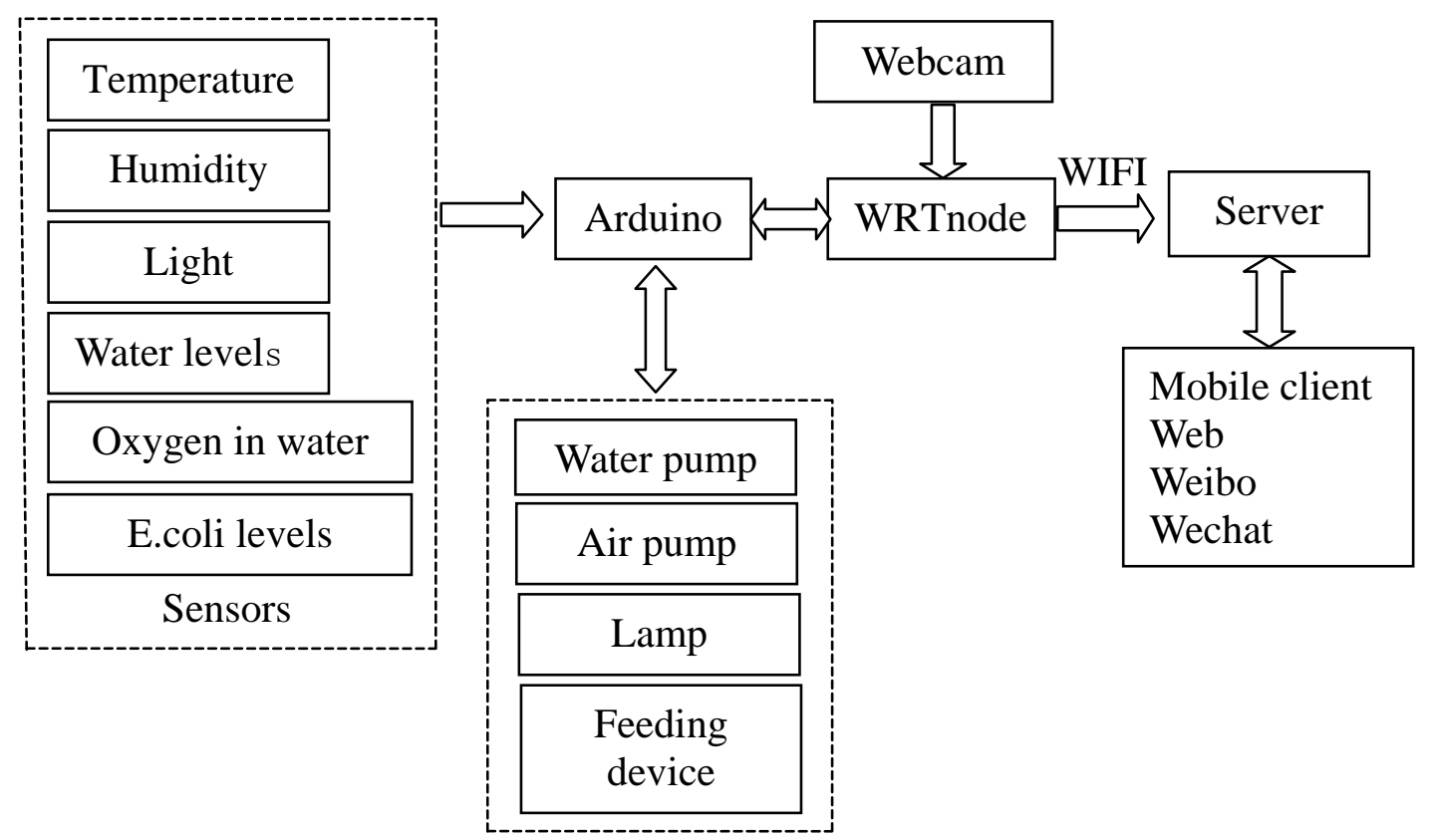

Fig.2. Flowchart of the hardware design of the system

\section{Arduino}

Arduino is an open-source physical computing platform based on a simple microcontroller board and a development environment that implements the Processing language ${ }^{[8]}$. Projects done with Arduino can be stand-alone or they can communicate with software running on a computer. Our design needs to be interactive, so it can make it much easier to create an environment in which learning can be achieved by doing, receiving feedback and refining understanding and building new knowledge.

\section{WRTnode}

WRTnode, based on Wi-Fi AP-Soc, is open source development board hardware ${ }^{[9]}$. It has many advantages. For example, it is mini, cheap and has pretty low power consumption and reasonable capability of computing. In addition, it is born to own high speed of Wi-Fi network exchange. In our project, it considers as a high speed choice. It can connect and interact with an USB device like camera and sound card, so we can collect images and voices at the same time and stream out multimedia stream in real time. After a layer of A/D converter, it can directly interact with physical world like all kinds of sensors and motors.

\section{Sensor nodes}

In this system, some sensors are used with different functions. Take the temperature and humidity sensors for example. This system adopts the DHT11 digital temperature and humidity sensor as signal acquisition unit, which has a free debugging, calibration free and the measurement resolution can be programmed to produce the ultra small package size. The sensor and integrated temperature and humidity sensors, humidity measurement operation can be used for temperature compensation, and can provide high quality of dew point calculation function, high measuring accuracy, is preferred equipment ${ }^{[10]}$ a variety of temperature and humidity testing applications. The typical application circuit diagram is shown in Fig. 3. 


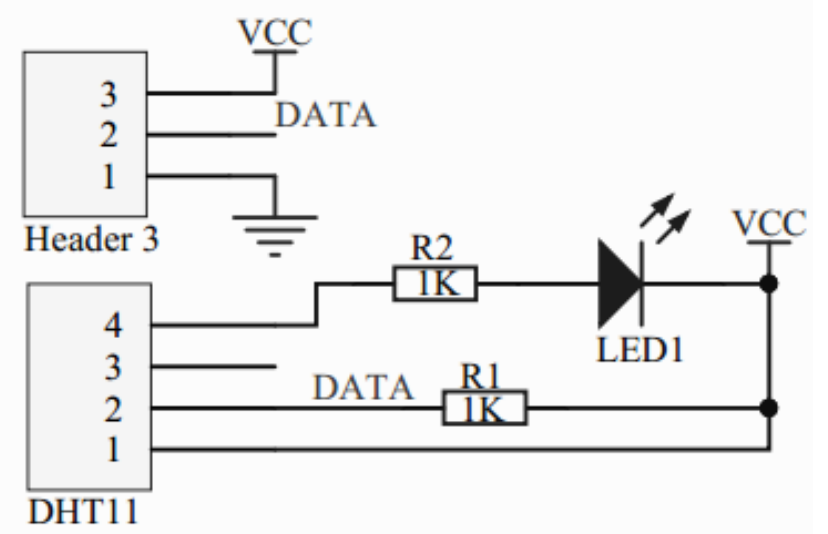

Fig.3. The typical application circuit diagram of DHT11

\section{Software design of the system}

OpenWrt, a distribution of embedded Linux ${ }^{[11]}$, is the system of WRTnode. When multimedia information and various input and output control focus on a Linux system, which has small volume, low power consumption and enough computing capacity, after porting and optimizing the existing pattern recognition, artificial intelligence and other applications, we may set up a series of interesting "smart" devices. They are supplemented by powerful network capabilities, and we may get a structure of MATRIX and NODE. OpenWrt buildroot source tree is shown in Fig.4.

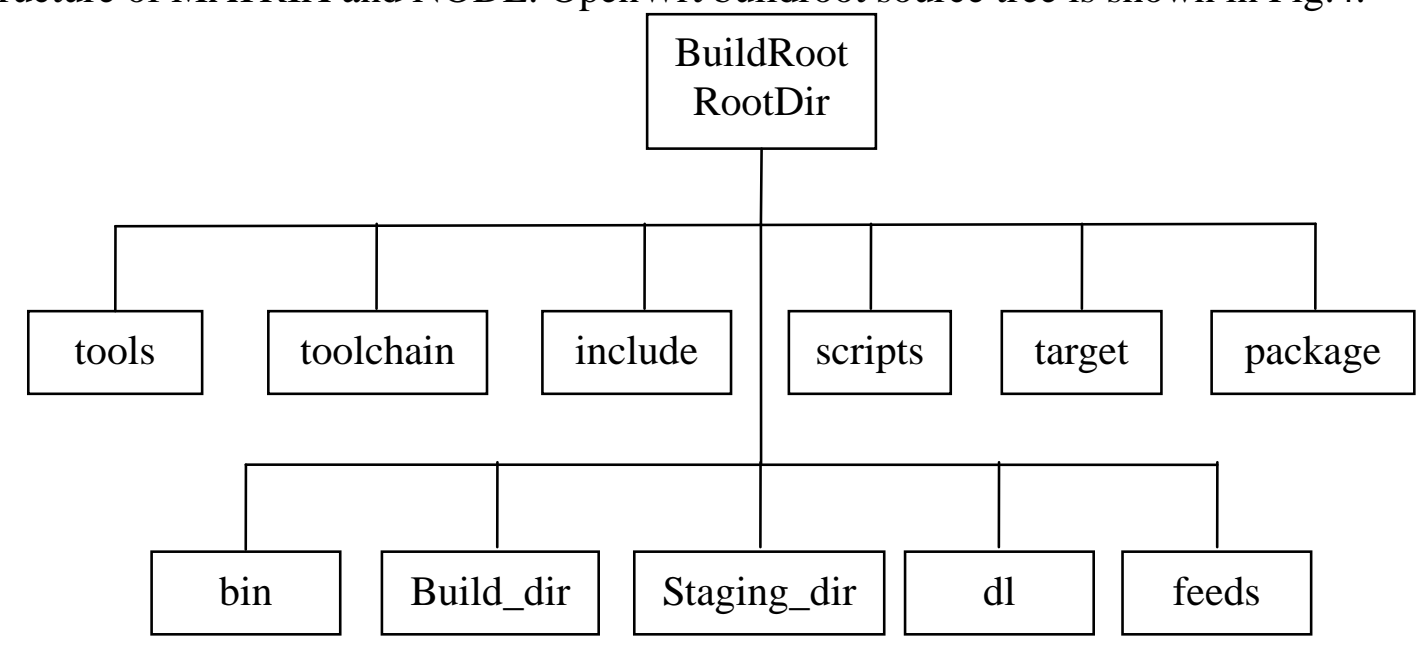

Fig.4. OpenWrt buildroot source tree

The first line is the original directory, and the second line directory is built during compiling. The function of the directory is:

tools - Containing the acquisition and compilation tools command, which are needed while compiling.

toolchain - Containing a number of commands to get kernel headers, C library, bin-utils, compiler, debugger.

target - The platforms in this directory defines a compilation of firmware and kernel.

package - The package contains Makefile for each package.

include - The makefile of OpenWRT are stored here.

scripts - Some of perl scripts for the package management.

$\mathrm{dl}$ - The packages are placed in this directory when they are download.

build_dir - The packages are extracted to build_dir.

staging_dir - This is the final installation directory, where tools, toolchain and rootfs are installed.

bin - The firmware and all ipk will be put in directory after the compilation is completed.

This study generates the OpenWRT SDK through compiling by GCC or Automaker when we get 
OpenWRT source code from SVN(Subversion) source.

FSWebcam gets original image from USB camera by a JPEG encoding, which are stored in WRTnode initial Flash. Then, the image files are sent to the HTTP server through the curl.

Data communicating with server is written in Python. Program uses reques package by JSON format string exchanging data, with server, sends the sensor data and obtains switch status value, according to the specified server API. At the same time, the program exchanges data with the Arduino through the serial for ensuring the data accuracy by CRC check, which enables indirect communications between the Arduino and the server. The communication part of the code is is as follows.

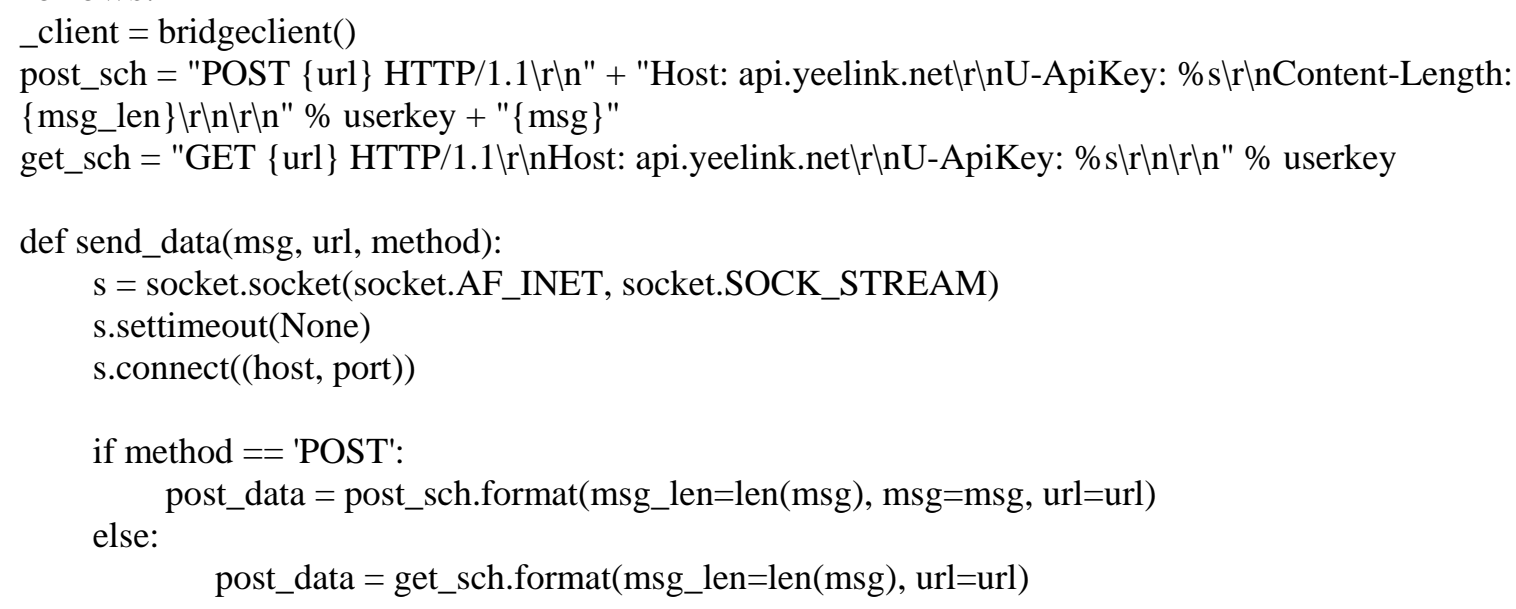

\section{Testing results}

The test application shows that it can receive the data from sensors timely and make a proper control of the appliances (Fig. 5), which indicates that the designed system has realized the desired functions. Users can see the detail information of humidity, light intensity, water level, photos, oxygen in water, E.coil levels. In addition, users can make a decision whether or not to turn on the air pump, water pump and lamp remotely according to the acquired information.

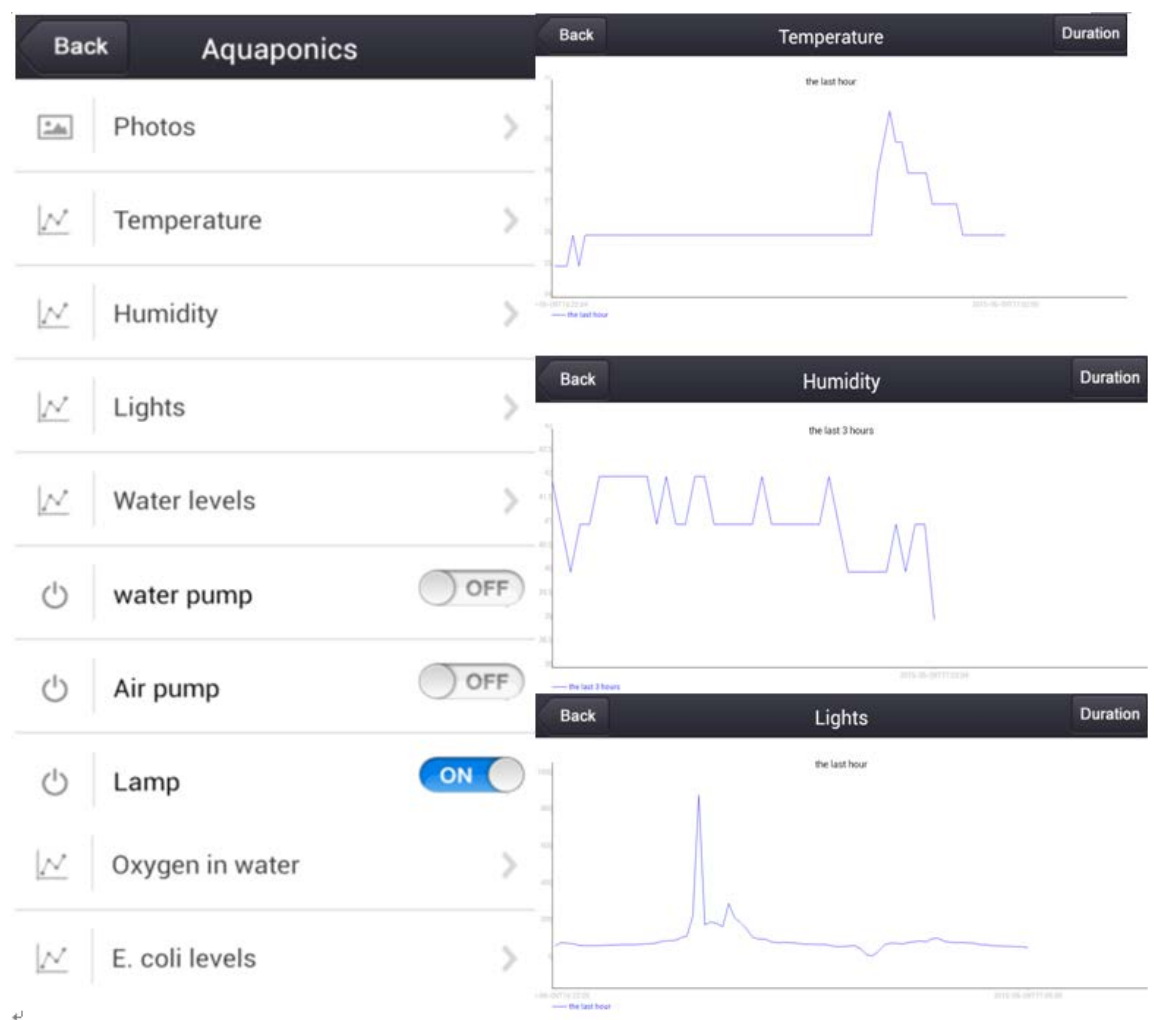

Fig.5. The monitoring data 


\section{Conclusion}

This study designs a smart monitoring and control system for aquaponics based on OpenWrt, which is intelligent, accurate and real time and can release the users from the hard working conditions. As one of typical applications, more and more people realize the application of the IoT (Internet of Things) will bring broad development to the smart life. Meanwhile, we have an idea that the device of aquaponics can be connected with social communication platform, which can realize a wonderful dream that people can interact with their ornamental fish and hydroponic plants on line through a mobile terminal.

\section{Acknowledgement}

The work was supported by the Leadership Introduction Project of Academy and Technology of Anhui University (No. 10117700024) and the Undergraduate Innovation and Entrepreneurship Training Program of Anhui University (No. 201510357393).

\section{References}

[1] Love D C, Fry J P, Genello L, et al. An international survey of Aquaponics Practitioners [J]. Plos One, 2014, 9: e102662.

[2] Fitter A H. The topology and geometry of plant root systems: influence of watering rate on root system topology in Trifolium pratense. Annals of Botany, 1986, 58(1): 91-101.

[3] Chen F, He H, Tang Y. In-situ optimal control of nutrient solution for soilless cultivation [C]. International Conference on Proceedings of the Advanced Computer Control (ICACC), 2011, 412-416.

[4] Shafahi M, Woolston D. Aquaponics: A sustainable food production system [C]. ASME 2014 International Mechanical Engineering Congress and Exposition, 2014, V003T03A073.

[5] Youquan Huang. Control system design is engaged in pisciculture to the intellect [J]. Development \& Innovation of Machinery \& Electrical Products, 2004, 2: 89-90.

[6] Zhai Yanni, Chen Xiaodong. Design of smart home remote monitoring system based on embedded system [C]. IEEE International Conference on Computing, Control and Industrial Engineering (CCIE), 2011, 41-44.

[7] Blidariu F, Grozea A. Increasing the economical efficiency and sustainability of indoor fish farming by means of aquaponics - review [J]. Animal Science and Biotechnologies, 2011, 44(2): $1-8$.

[8] Bender P, Kussmann K. Arduino based projects in the computer science capstone course [J]. Journal of Computing Sciences in Colleges, 2012, 27(5): 152-157.

[9] Gabriele S, Di Giamberardino P. A unified approach for heterogeneity and node fault robustness in dynamic sensor networks [J]. WSEAS Transactions on Communications, 2008, 7(7): 685-694.

[10] Bao X., Dhliwayo J, Heron N, et al. Experimental and theoretical studies on a distributed temperature sensor based on Brillouin scattering [J]. Journal of Lightwave Technology, 1995, 13(7): 1340-1348.

[11] Kim C G, Kim K J. Implementation of a cost-effective home lighting control system on embedded Linux with OpenWrt [J]. Personal \& Ubiquitous Computing, 2014, 18(3): 535-542. 